\title{
Grundsätzliches über grammatische Begriffe und grammatische
} Termini

Ein grammatischer Terminus ist ein $N$ a $m$ e für einen grammatischen Begriff. Man wählt ober bildet einen solchen Namen, d.h. einen Terminus, um einen grammatischen $B$ e $g r i f E$, den man sich erarbeitet hat, $f$ e $s t z u$ h a 1 t e $n$ - um mit dem Begriff arbeiten $z u$ können (selber) und um den Begriff andern zugänglich machen zu können. Durch das Aussprechen oder das bloße Vorstellen des Namens kann man auch den Begriff jederzeit in Erinnerung rufen, für sich selbst wie für andere Menschen; man kann durch das Nennen (oder rein durch das innerliche Vorstellen) des Namens den Begriff für einen Augenblick in den Mittelpunkt der Aufmerksamkeit rücken.

\section{Die Schaffung von Begriffen}

Der $\mathrm{B}$ e $\mathrm{g} r \mathrm{i} f \mathrm{f}$ e $1 \mathrm{~b} s \mathrm{t}$ wird durch die Namengebung nicht etwa geschaffen, sondern nur festgehalten. Der Begriff - als gedankliche GröBe, als "intellektueller Zugriff" - entspringt einem originären gedanklichen Akt, einer Einsicht auf Grund von Beobachtungen (meist auch schon geleitet von bestimmten Erwartungen, bestimmten Vorannahmen auf Grund einer Theorie). Die Beobachtungen, die zu grammatischen Begriffen führen, können spontan erfolgen (z.B. beim Hören oder Lesen von Texten), oder sie können im Zug systematisch durchgeführter operationen erfolgen, z.B. bei Ersatzproben, Verschiebeproben usw.

Aus diesen Einsichten, diesen intellektuellen Akten, ergibt sich nun jeweils eine $D$ e $f$ i $n i t$ o $n$ für den Begriff (auch wenn diese manchmal nicht explizit, sondern nur gewissermassen unterschwellig vorhanden ist).

2. Demonstration an zwei elementaren Satzgliedbegriffen Ein Beispiel: Man stellt fest, daß man aus Sätzen wie Jetzt 
hatte der zähe Alte doch endlich sein ziel erreicht die verbale wortkette herauslösen kann jetzt doch endlich sein $z i e l$ erreicht haben oder stärker vereinfacht jetat endiich sein $2 i e l$ erreichen. Der Ausdruck der ähe Alte läBt sich in diese verbale Wortkette im Infinitiv nicht integrieren, sondern steht ihr als etwas Eigenes gegenüber. Zugleich ergibt eine Verschiebeprobe, daß der a ähe Alte nur geschlossen verschiebbar ist 1 Der zähe Alte hatte jetzt doch endlich sein ziel erreicht), daB also ein $s$ a $t z \mathrm{~g} l \mathrm{i}$ e d vorliegt. Ebenso stellt man fest, daB der ähe Alte ein Nominalgefüge im Nominativ Singular ist und dab es nur durch andere nominale Ausdrücke oder durch einfache Pronomen im Nominativ singular ersetzbar ist $($ dieser Mensch er usw.) oder dann durch einen ganzen Nebensatz ( Der sich so lange umsonst abgemulht hatte, hatte jetzt doch endiich sein ziel erreicht). Schlieblich stellt man fest, daB bei einer Umformung des Ausdrucks in den Plural auch die Verb-Personalform (das. Finitum) in den plural gesetzt werden mus ( Jetzt $h$ a $t$ t $e n$ $d i e b e i d e n z a ̈ h e n A l t e n$ doch endlich ihr ziel erreicht).

Aus allen diesen Operationen und Beobachtungen ergibt sich nun der $B$ e $g$ i f $f$ :

Satzglied aus Nomen oder Pronomen oder ganzem Nominalgefüge im Nominativ, nur durch Nominativ ersetzbar oder dann durch ganzen Nebensatz levtl. nur Infinitiv), in singular/Plural mit der verb-Personalform übereinstimmend, beim Herauslösen der verbalen Wortkette im Infinitiv nicht in diese wortkette integrierbar, sondern als eigene Einheit daneben tretend.

Und für diesen B e g r i f f - jeder Leser des Bisherigen wartet wohl schon ungeduldig darauf - verwendet man nun den Te $r m i$ n u s (den Namen, den Fachausdruck) "Subjekt" oder "nominativus verbi, nominativus ante verbum" (Deutschland ${ }^{1}, 18$. Jahrhundert) oder "suppositum" (so der französische Humanist Petrus Ramus ${ }^{2}$, 1515-1572) oder "Satzgegenstand" (so die deutsche schultradition) oder "GrundgröBe" (so Glinz ${ }^{3}$ 1952) oder "E ${ }_{0}$ ) (so Engel 4 1982 ). 
Im zuge weiterer Operationen stellt man nun fest, daB bei den Verben oein/werden/bleiben/heiBen/genannt werden noch ein weiteres Satzglied im Nominativ vorhanden ist und daB dieses Satzglied in der verbalen Wortkette im Infinitiv bleibt, wenn man sie herauslöst: Ein Riese war er gerade nicht führt zu nicht gerade ein Riese sein/er. Für dieses satzglied mit der Definition "Nominativ in einer verbalen Wortkette mit sein/werden/ bleiben/heiBen/genannt werden. neben einem als subjekt dienenden Nominativ möglich, oft ebenfalls Kongruenz zur Verb-Personalform aufweisend" verwendet man nun den Terminus "Prädikatsnominativ" oder "Aussagenennwort" oder "nominativus post verbum" oder "Gleichgröße" (so Glinz 1952) ${ }^{6}$, oder "Gleichsetzungsnominativ" (Grebe 7 1959) oder "E7" (Engel ${ }^{8}$ 1982) oder möglichst kurz und neutral "Prädikativ" (Glinz" seit 1971). Nur am Rande sei vermerkt, daß für diesen wichtigen satzgliedbegriff in der KMK-Liste (unter Punkt 3, Satzlehre) überhaupt kein Terminus angegeben wird.

\section{Terminus als Beleuchtung des Begriffs}

Das Beispiel zeigt, wie häufig für e $i \mathrm{n}$ u $n$ d $d$ e $n$ s e l b e $n$ B e $g r$ i f $f$ ganz $v$ e $s c h$ e de $n$ e $T$ e $r m i n i$ gebildet bzw. gewählt wurden und ggf. nebeneinander im Gebrauch sind. Es handelt sich dabei gar nicht um verschiedene Begriffe, sondern nur um verschiedene Termini für ein und denselben Begriff lobwohl man, sogar von Linguisten, hie und da hört und liest "die deutschen Begriffe, die lateinischen Begriffe", wo eindeutig Termini gemeint sind). Sicher dienen die verschiedenen Termini oft zu einer bestimmten $B$ e $l$ e $u c h$ $t u g$ des betreffenden Begriffs, sie enthalten so etwas wie einen "Erklärungs-Ansatz" - aber sie bleiben trotzdem Termini, Namen, und sind nicht mit den Begriffen zu verwechseln. 
4. Beweglichkeit in der Handhabung verschiedener Termini für gleiche Begriffe

Das Beispiel zeigt auch: Je sicherer man einen Begriff beherrscht, je selbstverständlicher man mit den Identifikationsoperationen $f u ̈ r$ den Begriff umgehen kann, desto weniger wichtig wird der Terminus, desto leichter kann man bei Bedarf von einem Terminus zu einem andern übergehen.

In dieser bequemen Lage, daß man nämlich den Begriff schon völlig klar im Kopf hat und sicher beherrscht, ist man allerdings am Anfang der Arbeit meistens noch nicht. Das gilt nicht nur für den Laien, z.B. einen Schüler, der grammatische Begriffe lernen möchte/sollte, und es gilt auch nicht nur für den Linguisten, der die Ergebnisse anderer Linguisten kritisch überprüfen und bei Bestätigung übernehmen möchte/sollte; es gilt auch und besonders für den Linguisten, der einen bestimmten Bereich selber begrifflich durcharingen möchte, selber geeignete grammatische Begriffe entwickeln muB/will.

\section{Termini als Hilfen für die Begriffsentwicklung}

In der oben geschilderten Lage, beim Lernen oder beim erstmaligen Schaffen grammatischer Begriffe, kann ein geeigneter Terminus sehr wichtig und hilfreich sein - und ein ungeeigneter Terminus kann sich als sehr hemmend und ggf. ausgesprochen irreführend erweisen. Das gilt ganz besonders für den Linguisten, der neue Begriffe schaffen muB/will (weil die vorhandenen sich ihm als ungeeignet erweisen beim Umgang mit den Phänomenen) oder der aus diesen Grunde schon vorhandene Begriffe neu ordnen, auf eine neue Art zueinander in Beziehung setzen muB/will. Bei dieser Arbeit kann es nämlich besonders nützlich sein, wenn sich Termini bilden lassen (besser gesagt: wenn man sich eine Reihe von Termini einfallen lassen kann), die je e $i \mathrm{n}$ e g ee $i g n$ e $t$ e $M$ e $r k$ h $l$ f e enthalten und die einem dadurch helfen, sich die betreffenden Begriffe immer wieder in der geeigneten Beleuchtung und zusammengehörigkeit präsent zu machen, sie immer wieder in diesen konfigurationen aufzurufen. 
Ein geeigneter Terminus kann dazu beitragen, den einmal gewonnenen Blick auf die Phänomene, die einmal erreichte gedankliche ordnung besser festzuhalten.

Hier gerät man natürlich auch immer wieder in die Gefahr, daß man den Terminus überdeutet, ihn mit dem Begriff selbst verwechselt - wenn nicht beim begriffsschaffenden Linguisten selbst, so doch nicht selten bei den andern, die sich mit einer neugeschaffenen Begriffsordnung und der zugehörigen Terminologie auseinandersetzen.

Beispiel aus der eigenen Arbeit an den Satzgliedbegriffen für das Deutsche Ich darf hier vielleicht gerade etwas aus meiner eigenen Arbeit bei der Entwicklung der Begriffe für die fallbestimmten Satzglieder erzählen - in diese Reihe der fallbestimmten satzglieder gehören ja auch das subjekt und die Prädikative (d.h. das Prädikativ bei sein/werden/bleiben/heiBen/genannt werden und das Prädikativ zum objekt bei nennen, heiBen).

Der Nominativ neben dem subjekt war nämlich in der deutschen grammatischen Tradition lange zeit gar nicht als eigenes satzglied anerkannt (im Gegensatz zur französischen Grammatik, wo das "attribut du sujet", z.B. Il est $m o n$ a $m i$ schon lange als eigenes satzglied betrachtet wurde). Im Deutschen verstand man den Nominativ neben dem Subjekt, wie wohl die meisten heutigen Linguisten noch in der schule gelernt haben, nur als einen Teil des "prädikats", und mit dem Terminus "Prädikativ" meinte man damals sowohl den Ausdruck im Nominativ wie ein unflektiertes Adjektiv, das durch gein/ werden usw. mit dem subjekt verbunden ist - also ebenso $E r$ war ein kräftiger Junge wie Er war kräftig. Die satzgliedtheorie stand insgesamt quer zu den Wortarten. So konnte z.B.ein fallbestimmter Ausdruck wie das Haus (gleiche Lautung fur Nominativ und Akkusativ) in sechs verschiedenen Satzgliedfunktionen auftreten:
als Subjekt
als Akkusativobjekt
Dort steht d a s $H$ a $u$ s.
Du siehst $d$ a $s$ H a $u$ s.
als Präpositionalobjekt Er achtet $a u f d a s \quad H a u s$.
als Adverbiale des ortes $E r$ geht $a$ u $f$ d $a$ s $H$ a $u$ s $z u$.
als (präpositionales) Er genieBt den Blick a $u f d a s$ Attribut
als Prädikativ
$H$ a $u$ s.
Das weiBe Gebäude ist $d$ a s $H$ a $u$ s.

Mein erster schritt zu einer andern, befriedigenderen begrifflichen ordnung war nun, daß ich den mit "Attribut" gekennzeichneten Begriff aufgab und feststellte, jedes fallbestimnte satzglied (und manches fallfremde Satzglied) könne neben einem Kern oder Grundteil auch attributive Bestandteile enthalten. Mein Begriff "Satzglied" war dabei klar operational fundiert als "Wort oder Wortblock, der sich verschieben, aber nur geschlossen verschieben läßt, meistens um die finite Verform als 'Achse' herum".

Entsprechend beschränkte ich die Furktion "Strukturkern des Satzes", die man in der Tradition dem Prädikat+Subjekt zuschrieb, auf das $v e r b$ in seinen verschiedenen Teilen; dabei unterschied ich deutlich (auf Grund von verschiebeproben) zwischen der finiten verbform oder verb-Personalform und den zwei direkt damit verknüpfbaren infiniten Verbteilen, dem Infinitiv und 
dem Partizip II (damals "Partizip Perfekt" genannt). In diesen Funktionen der "verbalen Teile" sah ich nun nicht nur die Vollverben, sondern auch die "Hilfsverben" - denn fïr sie galten die stellungsregularitäten, die ich im Anschluß an Drach (1937) durch die Verschiebeprobe nachwies, in genau gleicher Weíse wie fir die "Vollverben". Dazu bildetet ich für die nichtverbalen Bestandteile von "trennbaren Verben" (also an in anfangen - wir fangen sofort an usw.l einen besonderen Begriff auf der stufe des Satzbaus, nicht auf der stufe der Wortbildung, nämlich: nichtverbales stijck, das eng mit einem Verb verbunden ist und das mit dem Infinitiv, den Partizipien und der nachgestellten Verb-Personalform zusammengeschrieben wird, bei Zweitstellung der Verb-Personalform jedoch als eigener Bestandteil des Satzes auftritt und den Endplatz beanspruchen kann, soweit dieser nicht von einer direkt verbalen Form besetzt ist. Als Terminus für diesen Begriff bildete ich "Verbzusatz" (Begriff wie Terminus haben sich dann sehr rasch durchgesetzt).

Bei den nichtverbalen Ausdrüken, die sich um die Verb-Personalform herum oder unter sich verschieben lassen (also den "Satzgliedern" in meiner Begriffsfassung - sie hat sich heute weitherum durchgesetzt) unterschied ich nun, in streng strukturalistischer Weise, nach dem Kriterium, ob diese Ausdrïcke $n u r$ a $u$ s $\mathrm{a} 1 \mathrm{l}$ e $\mathrm{s} t \mathrm{i} m \mathrm{~m}$ e $n$ Wörtern bestanden, ob fallbestimmte und fallfremde wörter $k \circ \mathrm{m} b$ i $i$ e $r$ waren (in allen Präpositionalausdrücken, meinen heutigen "Präpokasus") oder ob $\mathbf{n} \mathbf{r}$ f a 1 l $r$ e m d e wörter vorhanden waren.

Bei den rein fallbestimmten satzgliedern (ohne präpositionen) unterschied ich, je nach der möglichen Ersatzreihe:

- Subjekt (nur durch Nominativ oder durch Infinitiv oder durch ganzen Teilsatz ersetzbar)

- Prädikativ (nur durch Nominativ oder Teilsatz, kaum durch Infinitiv, oft aber durch fallfremdes wort ersetzbar, nämlich durch ein "undekliniertes Adjektiv")

- objekte im Akkusativ, Dativ, Genitiv (nur durch Ausdrücke im gleichen Fall ersetzbar)

- Adverbialakkusativ und Adverbialgenitiv (nicht durch Pronomen im Akkusativ bzw. im Genitiv ersetzbar, dafïr oft durch fallfremden Ausdruck)

Zum Prädikativ (das sich auf das subjekt bezog) kam dann noch das seltene Prädikativ zum Akkusativobjekt (Sie nannten $i h n d e n$ w $i$ a $e$ usw.).

Diese ganze Begriffsentwicklung wäre nun nicht möglich gewesen, wenn ich nicht immer wieder meine vorläufigen und dann dauernderen Ergebnisse terminologisch festgehalten, d.h. laufend neue, mir als Merkhilfen geeignet scheinende Termini gebildet hätte. Ich experimentierte also nicht nur mit meinen Probetexten und meinen Begriffsentwirfen, sondern auch mit allen möglichen Neubildungen von Termini - d.h. ich e $x$ p e $r i m e n t i e r t e$ auch mit den $\mathrm{N}$ a $\mathrm{m}$ e $\mathrm{n}$, die ich meinen $\mathrm{z} . \mathrm{T}$. Übernommenen, $z . T$. neu entworfenen Begriffen gab.

Ich will und kann das hier nicht vollständig vorführen; ich gebe nur einige Proben, die zeigen mögen, wie ich meine Termini immer wieder durch neue ersetzte - weil die Termini ja keinerlei Eigenwert hatten und haben sollten, sondern nur die Arbeit mit den Begriffen unterstützen sollten.

Eine erste Reihe (1944, in meinen Begriffs-Kennzeichnungen von damals, mit Verwendung des gelehrten Ausdrucks "Declinabile" für das, was ich später "fallbestimmtes Wort" bzw. "fallbestimute Wortgruppe" nannte): 


\section{finites Verb}

Infinitiv und partizip II mit

finitem Verb verbunden

Declinabile im Nominativ als Ausgangspunkt des im Verb dargestelten Verhaltens (gekennzeichnet durch Kongruenzforderung mit dem Finitum)

Declinabile im Nominativ neben einem subjekt, oft durch

Indeclinabile exsetzbar

Declinabile im Akkusativ, nur durch Akkusativ ersetzbar, bei Umwandlung ins Passiv zum Subjekt werdend

Declinabile im Dativ, nur durch Dativ ersetzbar

Declinabile im Genitiv, durch dessen, deren ersetzbar
Kernglied

Kernergänzungen

Subjekt

Nennergänzung

vertauschbare zielergänzung

beiläufige zielergänzung

altertïmliche Zielergänzung

Später probierte ich für den Nominativ neben dem Subjekt, also für das heutige Prädikativ, den Terminus "Nennglied" gegeniiber den objekten als "Zielgliedern", nämlich einfaches "Zielglied" für das Akkusativobjekt, "Mitzielglied" für das Dativobjekt (ausgehend von den häufigen Kombinationen jemandem etwas geben/nehmen usw.) und "Anteilglied" für das Genitivobjekt. Fir das subjekt benïtzte ich dann, um die Zusammengehörigkeit in der Reihe sichtbar zu machen, den Terminus "Grundglied".

Im Blick auf das starke streben des prädikativs nach Endstellung hatte ich eine Weile in Analogie zum "Verzusatz" ( gab nach, hörte auf) fïr das Prädikativ den Terminus "zusatzfall" (dann waren analog nebeneinander der fallfremde "Verbzusatz" und der Nominativ neben einem Subjekt oder der Akkusativ neben einem Akkusativobjekt als "fallbestimmtes Satzglied mit Streben nach Endstellung", eben als "Zusatzfall"). Dann probierte ich mit "Einordnungsglied, Gleichsetzungsglied", wobei der Terminus nicht mehr das streben nach Endstellung, sondern vor allem den semantischen Charakter festhalten sollte.

Fï die ganze Gruppe der fallbestimmten Satzglieder kam ich dann auf den Terminus "Größen" (wegen der Möglichkeit, sie im Singular oder im Plural zu brauchen). Für das Prädikativ bildete ich "Einreihgröße" und schließlich das kürzere "Gleichgroße", bei dem ich dann lange zeit blieb. Für den festen Dativ (Dativobjekt mit Einschluß des "dativus ethicus") kam ich zur "Zuwendgröße" - einem Terminus, von dessen überdeutung durch Kollegen noch zu sprechen sein wird. Die fallfremden Satzglieder nannte ich "Angaben"; dieser Terminus wurde dann von jüngeren Linguisten für einen ganz anderen Begriff in Anspruch genomen, nämlich für die nicht-obligatorischen Satzglieder, in der bekannten Entgegensetzung von "Ergänzungen" und "Angaben". 
6. Betonung der Relativität und Dienerrolle aller Termini

Es geht wohl schon aus diesem knappen Bericht hervor, daB mir der Unterschied von Begriff und Terminus immer völlig klar war - so sehr ich versuchte, Termini mit "günstiger begriffsbeleuchtender Wirkung" zu finden; ich habe daher in der "Inneren Form des Deutschen" immer wieder ausdrücklich und nachhaltig auf den Unterschied von Begriff und Terminus und auf die bloBe Dienerrolle der Termini aufmerksam gemacht. Bei der ganzen Arbeit und Darstellungsweise ging ich von der (wie sich bald zeigen sollte: $\mathrm{zu}$ optimistischen) Uberzeugung aus, jeder kritische Leser meines Buches werde diese klare Unterscheidung von Begriff und Terminus nachvollziehen. Ich bin auch heute noch überzeugt, dab man von jedem wissenschaftlichen Leser - und speziell von jedem Linguisten - verlangen kann und muB, daß er jederzeit einen ihm neuen Terminus auf Grund der Begriffsbestimmungen als Terminus durchschaut und $d a \beta$ er überhaupt im Bedarfsfall jederzeit einen Terminus durch einen anderen ersetzen kann (wenn er Publikationen von kollegen mit anderer Terminologie liest), ohne daB die Präzision der gedanklichen Arbeit, die Genauigkeit des Verstehens darunter leidet.

7. Uberschätzung von Termini bei Lesern, Uberinterpretationen

Die Praxis belehrte mich leider bald, daB die eben genannte Forderung gar nicht immer erfült wurde. Was ich als $T$ e $r$ m i$\mathrm{n} u$ s angeboten hatte, nach der Kennzeichnung des Begriffs durch Angabe der $\mathrm{zu}$ ihm führenden Operationen, wurde von manchen als $B$ e g r i f $f$ d e $f$ i $n i t i$ o $n$ verstanden, wurde mit dem Begriff gleichgesetzt - ja manchmal als ein Begriff genommen, den ich gar nicht angegeben hatte, sondern den der lesende und kritisierende Linguist dem Terminus zulegte.

Ein Beispiel für manche: Peter von Polenz (1969) kritisierte die von Leo Weisgerber gegebene charakteristik "zugewandter Betätigungssatz" (für den Satztyp Die Sonne schien ihm auf den Kopf ); im AnschluB an das Wort "zuwenden", das Weisgerber in 
dieser Charakteristik gebraucht hatte, macht nun Polenz die folgende Anmerkung (S.157):

"Der in der 'inhaltsbezogenen' Grammatik gern und vielfältig benutzte Terminus 'zuwenden' geht auf die generalisierende semantische Festlegung der Kasus in H. Glinz' kursorischer Kategorisierung zurick: "Zuwendgröße für das Glied im Dativ" (Innere Form ...., \$. 165), "Größe ... der sich etwas zuwendet" (ebda., S. 163). Vorbild für diesen Terminus waren die Verben des 'Gebens' und vielleicht die wörtlichgenommene antike Bezeichnung 'dativus', was aber auf die semantische Funktion des Dativs in unserem satzbauplan nicht zutrifft."

Dazu ist von mir aus zu sagen: Auf der zitierten Seite der "Inneren Form des Deutschen" wird von "Fallgliedern", d.h. von $\mathrm{s}$ a $t$ z g 1 i e d e $\mathrm{n}$ gesprochen, die durch einen ersatzfesten Fall gekennzeichnet sind - und keineswegs von den Fällen a $n$ s i c h. "Zuwendgröße" ist also in keiner weise eine generelle Charakteristik des Kasus "Dativ" (der ja oft auch in Verbindung mit einer präposition auftritt, nicht nur als Dativobjekt), sondern ein beleuchtender Terminus für das $s$ a $t z-$ g 1 i e d "Dativ o b j e $k t$ ". Das ist etwas ganz anderes als der $K$ a $s$ u $s$ "Dativ" (es sei denn, man verwende "Kasus" doppeldeutig, nämlich einmal formal für das, was man im Deutschen, Lateinischen und Griechischen "Fall" nennt und einmal im sinne von Fillmore als semantischen satzgliedbegriff oder "Tiefenkasus"). Ich habe diesen Gebrauch von "Zuwendgröße" wegen ähnlicher vorgekommener Mißverständnisse auch ausdrücklich von der 2 . Auflage (1961) an auf S. 14 der "Lesehilfe" durch "Objektsdativ" verdeutlicht. Die vier Kasus habe ich durch das ganze Buch hindurch mit den gewohnten lateinischen Namen "Nominativ, Akkusativ, Dativ, Genitiv" bezeichnet. Der beleuchtende Terminus "zuwendgröße" ist also nicht etwa aus dem Terminus "Dativ" (von dare, als Lehnübersetzung des griechischen dotike, herausgesponnen, wie Polenz es bei mir zu sehen glaubt, sondern er ist gewonnen aus der Beobachtung der sehr häufigen Kombinationen jemandem etwas geben/nehmen/ anbieten/mitteilen/ verweigern/ rauben usw; auch wenn jemand jemandem etwas raubt, muß er sich ihm und seiner Habe ja "zuwenden", ganz wörtlich. 
Die Uberinterpretation eines Terminus, seine Hochstilisierung zur Begrifisdefinition, kommt nicht nur bei Germanisten vor, wie das folgende (etwas komplizierte) Beispiel zeigt. Ein Aufsatz von P. Erdmann (1983) trägt den Titel "Zur 'Existenzialität' von there-sätzen im Englischen". Was ist hier 'Existenzialität'?

Konstruktionen vom Typ There is a problem, there are many probLems werden in der englischen Grammatik seit langem als "existential sentences" bezeichnet. Die Benennung geht offenbar auf Jespersen (1929) zurück, der allgemein zu derartigen Konstruktionen (nicht nur im Englischen) schreibt (zitiert nach Erdmann ):

Sentences corresponding to English sentences with there is or there are, in which the existence of something is asserted or denied -if we want a term, we may call them existential sentences - present some striking particularities in many languages.

Bemerkenswert ist hier die Vorsicht bei der Einführung eines Terminus und die klare Unterscheidung zwischen Terminus und Begriff.Jespersen gibt zuerst eine Beschreibung des Begriffs und betont dann den spielraum bei der wahl einer Benennung (die "Arbitrarität", ob man für die betreffende Erscheinung einen Terminus haben will und was für einen), indem er sagt " - if we want a term, we may call them...."

Um einiges kategorischer klingt dann die Darstellung von Quirk/ Greenbaum (1973,S. 418):

\section{Existentisl centences \\ 14.19}

Existential sentences are principally those beginning with the unstressed word there, and are so called because when unstressed there is followed by a form of the verb be, the clause expresses the notion of existence:

There is nothing healthier than a cold shower

('Nothing healthier exists than a cold shower')

Andere Grammatiker verwenden keinen besonderen Begriff für die there is... -Konstruktionen und daher auch keinen Terminus "existential sentences/clauses", auch wenn sie für die charakteristik der Bedeutung von to be das Wort "existence" benützen so Thomson/Martinet, (1970, S. 66): 


\section{'To be' used as an Ordinary Verb}

112 To denote existence

a to be is the verb normally used to denote the existence of, or to give information about, a person or thing:

Tom is a corpenter. The dog le In the garden,

Malta le an laland. The roada were rough and narrow.

Gold le a metal.

Peter was tall and falr.

b there $b$, there are

When a noun representing an indefinite person or thing is the subject of the verb to be, we usually put there before the verb and the noun after it:
II /s posalble to say
But il is more usual to say
A man Ia In the hall.
Simllarly we can say

\begin{abstract}
There le man In the hall.
\end{abstract}
There la an egg In that neat.
There are egoe in that neat.

Interessant für das Thema "Begriff und Terminus" sind nun die drei folgenden Stellen aus dem Aufsatz von Erdmann (1983):

In diesem Beitrag möchte ich drei Punkte wieder aufgreifen, die in einer friheren Arbeit zu diesem Problem angesprochen wurden (Erdmann 1976) und die dazu beitragen, einige Gesichtspunkte der möglichen "Existentialität" von there- Sätzen zu präzisieren. Es sind dies 1. die Quantifikation ihrer subjekte. 2. das Erscheinen bzw. Nicht-Erscheinen von prädikativen Nomina und 3. das Auftreten von verben außer be in thereSätzen. (S. $20-221$ )

Die Beispiele lassen sich dahingehend zusammenfassen, daß in thereSätzen nur solche quantifizierenden Ausdrücke erscheinen dirfen, die einen Gegenstandsbereich eingrenzen. Spricht man von der Existentialität von there- Sätzen, so ist damit die Besonderheit gemeint, daß in thereSätzen nur linguistische Existenz- aber keine linguistischen Allquantoren auftreten können (vgl. hierzu Milwark 1977: 4-9); Hogg 1977: 63f.) (S. 223)

Die englischen there- sätze zeigen zweitens eine eigentiumliche Restriktion im Hinblick auf die Adjektive und substantive, die in ihnen erscheinen können. Auch diese Besonderheit, die sowohl eine lexikalische wie syntaktische seite hat, dient zur Aufhellung des Begriffs der "Existentialität".

Ein solches Ernstnehmen des Wortes "existence" im Terminus ist unbedenklich, ja begrüBenswert, solange klar die Frage gestellt wird: Wie weit ist die Bezeichnung dieser Konstruktionen als "Existenzsätze" den beobachtbaren phänomenen angemessen, wie weit liefert sie eine $n$ ü $t z l$ i $c h$ e $B$ e $l$ e u c h $t$ u $n g$ für den Begriff "clauses, die mit there $i s$, there are usw. anfangen"? Dagegen kann es gefährlich werden, wenn ein besonderer Begriff "Existenzialität von Sätzen (bzw. von clauses)" aufgebaut wird und wenn dann diese "Existenzialität" als gemeinsamer 
begrifflicher Faktor aufgefaßt wird in den doch recht verschiedenen Begriffen, die man durch die Termini "existential sentences" und "Existenzquantoren" bezeichnet.

9. Verschiedene Subjektbegriffe englischer Grammatiker und zugehörige Termini

Die Stellen über die "Existenzsätze" gestatten zugleich die Beobachtung, daß führende englische Grammatiker der Gegenwart in der Anwendung ganz elementarer grammatischer Begriffe bei gleichen Phänomenen $z u$ verschiedener Analyse und damit natürlich auch $z u$ verschiedener Benennung von Bestandstücken gleicher Konstruktionen kommen. Bei Quirk/Greenbaum (1973) liest man auf S. $419-420$ :

14.20

Existential there as subject

The there of existential sentences differs from there as an introductory adverb both in lacking stress, and in behaving in most ways like the subject of the clause:

(a) It often determines concord, governing a singular form of the verb (especially in declarative sentences) even when the following 'notional subject' is plural (see 7.18 Note $a$ ):

There's two patients in the waiting room (informal) occurs alongside:

There are fwo patients in the waiting room

(b) It can act as subject in yes-no and tag questions:

Is there any more soup?

There's nothing wrong, is there?

(c) It can act as subject in infinitive and -ing clauses:

I don't want therc to be any misunderstanding

He was disappointed at there being so litile to do

Auf der vorhergehenden seite (418) findet sich eine differenziertere Darstellung, indem zwei Arten von "Subjekt" unterschieden werden, nämlich "notional subject" und "grammatical subject". Was in der "regular clause" subjekt ist, ohne Differenzierung, wird in der "existential clause" zum "notional subject", und neben diesem gilt there als "grammatical subject": 
may derive existential clauses from regular clauses by means of a general rule:

subject + (auxiliaries) + be + predication

$\rightarrow$ there + (auxiliaries) + be + subject + predication

The subject of the original clause may be called the 'notional' subject of the there-sentence, so as to distinguish it from there itself, which for most purposes is the 'grammatical' subject (14.20).

Diese Einführung von zwei verschiedenen subjekten (sie erinnert an das "logische subjekt" und das "grammatische subjekt" bei Hermann Paul) drängt sich für Quirk/Greenbaum offenbar auf, weil sie das subjekt nicht nur operational, sondern auch semantisch definieren: das subjekt wird als das typische Eröffnungsglied für die clause gesehen, es liefert das "Thema" für die in der clause dargestellte Aussage. Diese Rolle übernimmt nun in den there-Konstruktionen das there, und daher wird dieses als "grammatical subject" betrachtet; der dem there is... Eolgende nominale Ausdruck muß aber doch auch in gewissem sinn als Subjekt anerkannt werden, und daher erhält er nun den Namen "notional subject".

Eine ganz ähnliche Aufspaltung des Subjektsbegriffs in zwei Unterbegriffe findet sich bei F.S. Scott et. al.(1968, S. 187). Dort wird das there in solchen Konstruktionen als "substitute subject" bezeichnet; was bei Quirk/Greenbaum (1973) "notional subject" genannt wird, trägt bei scott den Namen "appositional subject" (dieser Name gilt auch für manche Konstruktionen mit it is..., scott 182). Auch hier steht im Hintergrund wohl die Auffassung, die stellung vor der Verb-Personalform sei die Normalstellung für ein subjekt - und das there, das in den there is -Konstruktionen diese stellung einnimmt, müsse daher auch so etwas wie ein subjekt sein.

Andere Grammatiker, die auf semantische Komponenten in der subjektsdefinition verzichten und ebenso auf die starre stellungsbindung, wie Thomson/Martinet 1979, akzeptieren dagegen auch den nach there ist..., there are ... folgenden nominalen Ausdruck als normales subjekt - vor allem auch, weil er die kongruenzforderung mit der verb-personalform, die für die subjekte typisch ist, erfüllt. Man liest bei Thomson/Martinet auf s. 67: 
Notice that, though there appears to be the subject, the real subject is the noun that follows the verb, and if this noun is plural the verb must be plural also:

There la a clgarette In that box. There are clgarettes In that box.

There has been atorm. There have been storms.

There was a queue at the station. There were queues at the station.

Diese Auffassung stimmt mit dem überein, was man allgemein in den Grammatiken indoeuropäischer sprachen als subjekt definiert, auch im Deutschen.

10. Kennzeichnung ad hoc oder neuer Terminus?

Das zitierte Beispiel mit "notional subject - appositional subject - substitute subject" kann zugleich ein problem sichtbar machen, das man in der Diskussion der Fachleute uber das Verhältnis von Begriff und Terminus gelegentlich vergiBt.

Für den Benutzer einer Grammatik ist nämlich gar nicht immer klar, ob eine Bezeichnung nur als charakteristik eines einzelnen Phänomens zu verstehen ist, ad hoc, oder ob ein fester, durchgehend gebrauchter Terminus anzunehmen ist. Diese Frage kann umso eher auftreten, als man Grammatiken ja gewöhnlich nicht von vorn bis hinten liest (wie man es mit Monographien tun sollte), sondern jeweils nur einzelne stellen nachschlägt.

So könnte ein terminologiefreudiger Benutzer annehmen, daB mit "real subject" bei Thomson/Martinet eben doch ein neuer Terminus geschaffen sei, und daß man dann so etwas wie "Realsubjekt" von einem "bloßen scheinsubjekt" unterscheiden müsse (in extensiver Auslegung des Nebensatzes "..., though there appears to be the subject"). Umgekehrt könnte man fragen, ob bei scott et.al. mit "substitute subject" wirklich ein eigener Begriff gemeint sei, eine besondere Unterart von Subjekten, oder nur etwas wie "Wort, das die sonst vom subjekt eingenommene stelle ausfüllt". Die gesamte präsentation, mit EinschluB der Registerstich- wörter, spricht allerdings eher gegen diese zweite, nichtterminologische Auffassung - das grundsätzliche Problem besteht aber hier auch (und vielleicht nicht nur für einen nicht-englischsprachigen Leser). 
11. Emotionale Aspekte, positiv: Befriedigung durch Ubernahme neuer Termini

Das ganze problem "Begriffe und Termini" hat nicht nur eine rein rationale, sondern auch eine emotionale Seite: neue Termini können Emotionen wecken, und zwar sowohl positive wie auch (und wohl häufiger, vor allem bei Nicht-Linguisten) negative Emotionen.

Ein Terminus oder eine ganze serie neuer Termini kann den Bedürfnissen und wünschen von Benutzern entgegenkommen, ihnen hilfreich erscheinen und daher gern übernommen werden, mit positiven Gefühlen. Es kann auch sein, daß eine neue Terminologie besonders "wissenschaftlich" aussieht, das sie daher denjenigen, die sie kennenlernen, als vorteilhaft für ihre wissenschaftliches "image" oder generell für ihr sozialprestige erscheint und daß sie aus diesem Grund übernommen wird, viel eher als aus rein sachlichen Bedürfnissen. Beispiele für eine manchmal überbordende verwendung neuer Termini aus eher sozialpsychologischen als aus rein sachlichen Gründen hat es gerade in der deutschsprachigen Linguistik und Grammatik seit den $60 \mathrm{er}$ Jahren zur Genüge gegeben. Durch die Verwendung neuer Termini konnte man das Gefühl haben, man sei "in", man marschiere in vorderster wissenschaftlicher Front - so wie die Verwendung bestimmter besonderer wörter (oder die vermeidung von als unattraktiv eingeschätzten wörtern) seit je der Förderung des Sozial-prestiges gedient hat.

12. Emotionale Aspekte, negativ: ärgerliche Ablehnung neuer Termini

Es können sich aber auch negative Emotionen einstellen. Dabei ist zu unterscheiden, ob neue Termini vorgeschlagen werden, weil neue Begriffe geschaffen oder jedenfalls bisherige Begriffe modifiziert worden sind, oder ob aus irgendwelchen Gründen bei gleichbleibenden Begriffen neue Termini propagiert werden. 
Neue Begriffe oder Änderungen an vorhandenen Begriffen verlangen vom Benutzer einen erhöhten geistigen Aufwand, sie verlangen ein Neu-Lernen oder ein Umlernen - und gerade das Umlernen kann jemandem schwerer fallen als das Neu-Lernen.

Ein Stück Umlernen wird aber auch verlangt, wenn (nur) ein neuer Terminus angeboten wird (etwa im $\mathrm{zug}$ von großräumigen terminologischen vereinbarungen, z.B. für schulen) und der Begriff genau gleich bleibt. In markantem Gegensatz zu den tatsächlichen Rangverhältnissen stellt man nämlich auch in solchen situationen immer wieder fest, daß rein terminologische Änderungen (der Ersatz eines vertrauten Terminus durch einen neuen, der z.B. für Anfänger, für kleine Schüler günstiger wäre, bei völlig gleich bleibendem Begriff) von manchen als störend, ja als ungehörig empfunden werden - auch und gerade von manchen Fachleuten.

13. Sprachpsychologische Gründe für das Festhalten an einem vertrauten Terminus

Das immer wieder beobachtbare widerstreben auch gegen rein terminologische Änderungen läßt sich zunächst recht einfach aus den Gesetzen der Arbeitsökonomie erklären: jedes Umstellen auf eine neue signalisierung von etwas, auch wenn das dadurch signalisierte völlig gleich bleibt, verlangt eine zusätzliche gedankliche Anstrengung und Gedächtnisleistung. Diese ist besonders hoch, wenn der neue Terminus in sich keine verstehens- und Merkhilfe bietet.So muß ich z.B., obwohl ich mich als linguistischen "Insider" betrachten kann, für die von U. Engel (1982) als E。 bis $E_{9}$ benannten Satzgliedbegriffe immer wieder in Engels Buch nachschlagen - weil ich nicht auswendig weiB, was nun E4, E7 usw. ist.

Die Wurzeln für das widerstreben gegen neue Termini und das Festhalten an den vertrauten liegen aber noch tiefer. Je schwerer nämlich das $E r w e r b$ e $n$ eines Begriffes ist, je mehr gedankliche Anstrengung man in diesen Erwerb investieren muB, umso mehr $h$ ä $n g t$ man am $\mathrm{T}$ e $\mathrm{r} m \mathrm{i} \mathrm{n} u \mathbf{s}$, den man bei diesem (ersten) Erwerb gelernt hat. Und auch wenn man den Begriff ohne 
Mühe handhaben kann, ist man oft an eine Terminologie so sehr gewöhnt, daß man eine Umstellung scheut, ja u.U. (was sachlich gar nicht zuzutreffen braucht) in der Umstellung der Termini eine Gefahr für die Klarheit der Begriffe sieht.

\section{Kleiner Exkurs zur Wichtigkeit bestimmter Namen, generell}

Man kann hier die Linien zurückverfolgen bis in die Wertschätzung des Namens einer sache oder vor allem einer Person in alten, magischen Zeiten. Wer den $\mathrm{N}$ a $m$ e $n$ von jemandem wußte (z.B. den Namen einer göttlichen Macht, von welcher er Hilfe begehrte), der konnte durch diesen Namen den $k$ $n t a k t$ mit der betreffenden Person, der betreffenden Macht herstellen, er hatte ein Stick $v$ e $r$ f i g u $n g s g$ e w a $l \mathrm{t}$ iber den, dessen Namen er wußte. Man kann hier an das Märchen vom Rumpelstilzchen erinnern, in welchem ja die Macht des bösen Rumpelstilzchens darin beruhte, daß niemand seinen Namen wußte; mit dem Aussprechen dieses Namens war auch die Macht Rumpelstilzchens gebrochen. Man kann an die Art erinnern, wie odysseus auf der Insel der Phäaken den Flußgott anfleht, dessen Namen er nicht weiß, von dem er aber erreichen möchte, daß ex die Fluten des Flusses hemmt: "Höre mich, Herrscher, wer du auch seist! Ein Rufender naht dir...". Man kann an die Chnmacht des geblendeten Kyklopen Polyphem erinnern, dem sich odysseus unter dem falschen Namen "Niemand" vorgestellt hat und der daher nun bei seinem Schreien "Mich überwältiget Niemands Trug ..." von den andern Kyklopen ausgelacht wird.

Man kann solche magische Vorstellungen von der Macht von Namen noch heute nachvollziehen (ganz abgesehen von der sehr rationalen List des odysseus mit seinem "Niemand"), wenn man an Telefonnummern denkt: wer die Nummer von jemandem weiß, kann diesen anrufen; und nichts ist ärgerlicher, als wenn man eine überholte Numer einstellt, umsonst auf eine Reaktion wartet und schließlich merken muß, daß der Partner aus irgend einem Grund eine andere Nummer bekommen hat.

Entsprechende Wichtigkeit eines ganz bestimmten Namens fïr etwas gab es, ganz abgesehen von Magie, auch im Rechtswesen. In den Institutionen des Gaius (IV/II) liest man von einer Klage wegen Beschädigung von weinstöcken: sie wurde abgewiesen (obwohl vermutlich in der sache berechtigt), weil der Kläger sich auf ein Gesetz "de vitibus succisis" ("von abgehauenen Weinstöcken") berufen hatte; nun gab es aber kein Gesetz mit diesem Wortlaut, sondern eines mit der Formulierung "de a $r$ b $r$ i b u $s$ succisis" (d.h. "von abgehauenen Bäumen"). Die "vites" wären unter den "arbores" zu zu subsumieren gewesen - aber die wahl des zwar geläufigeren und genaueren, aber rechtlich nicht vorgesehenen wortes führte zum Verlust des prozesses durch den Kläger. 
15. Beibehaltung eines Terminus auch bei Veränderung des Begriffs

Die oben beschriebene elementare wertschätzung eines eingeführten Namens kann es nun auch verständlich machen, dab verfasser von Grammatiken manchmal für einen neuen Begriff lieber den für die frühere Fassung dieses Begriffs vorhandenen, vertrauten Terminus beibehalten als für den neuen Begriff auch einen neuen Terminus $z u$ wählen. So ist z.B. "Prädikat" in der Duden-Grammatik seit 1959 etwas ganz anderes als bis 1935; das neue "Prädikat" umfaßt nur noch die verbalen Teile, d.h. Verbpersonalform, Infinitiv bzw. Partizip II und verbzusatz, nicht mehr wie früher auch die nominalen und adjektivischen Prädikatsteile (neben denen das Verb blobe "Kopula" war). Der B e $g r$ i f $f$ ist also verändert, vereinfacht, geklärt worden - der $\mathrm{T}$ e $\mathrm{r} \mathrm{m} \mathrm{n} u \mathrm{~s}$ ist beibehalten worden.

16. Der Terminus für den Laien: Durchsichtigkeit als Hilfe oder als Irreführung

Nun geht es bei den Referaten dieses Bandes ja nicht vor allem um den Gebrauch des Terminus durch die Fachleute, sondern um den Gebrauch durch die Laien, nämlich durch die $L$ e $h$ r e $r$ aller stufen und in allererster Linie durch die $s$ c h $\ddot{u} l$ e $r$. Es ist also zu fragen: $w i$ e $l$ e $r$ t jemand einen gramatischen Begriff, und inwiefern ist ihm dabei der Terminus eine $H$ i 1 f e oder (bei ungeeignetem Terminus) eine $s$ ö $r$ u $n g$ ?

Das Wesentliche zu diesen Fragen wird im Beitrag von Elly Glinz in diesem Band gesagt, und ich kann mich daher hier auf einige globale Feststellungen beschränken, nämlich:

a) $0 \mathrm{~h} n$ e Terminus geht es $n i c h t$. Als Besiegelung, zum Festhalten der Ergebnisse der gedanklichen Arbeit bei der Begriffsbildung braucht man ein sichtbares und hörbares signal, ein Wort, einen Namen, eben einen Terminus.

b) Man darf aber die Arbeit an der Begriffsbildung nicht etwa mit dem Terminus anfangen. Man muB anfangen mit $g$ e $l$ e $n k-$ t e $n$ B e b a c h $u^{\prime} \mathrm{g} e \mathrm{n}$, wenn immer möglich mit eigenen Operationen der Lernenden, schlicht gesagt: mit $\mathrm{P}$ I o - 
b $i$ e $r$ e $n$ von sprachlichen Möglichkeiten; dabei sind auch nichtverbale Mittel der Verdeutlichung (graphische Figuren, Farbkennzeichnungen) wertvoll, wenn man sie nicht überschätzt, sondern eben als Hilfen anbietet.

c) Auch im $A$ u f b a d e $s$ T e r m i n s $s$ e 1 b $s t$ kann eine Hilfe liegen, wenn nämlich ein geeigneter "durchsichtiger Terminus" vorhanden ist - oder es kann vom Terminus eine störung ausgehen, wenn er nämlich irreführende Assoziationen nahelegt.

Die Hilfe, die man sich von einem geeigneten "durchsichtigen" Terminus verspricht (d.h. von einem "motivierten" Terminus im Sinn von Saussures "relativement motivé") war der Grund für die schaffung so vieler "sprechender" Termini, auch und gerade in der deutschen Grammatik seit dem späten 18. Jahrhundert: "Satzgegenstand" für subjekt, "Aussage" für Prädikat, "Eigenschaftswort" oder "Beiwort" für Adjektiv usw.

Das streben nach Durchsichtigkeit galt aber auch bei der seinerzeitigen schaffung der lateinischen Termini, deren Vorteil heute oft gerade darin liegt, daB sie nicht (mehr) durchsichtig sind und daher als reine "Etiketten" ohne irreführende wörtliche Bedeutung dienen können. Für diejenigen, die sie vor zwei Jahrtausenden und mehr geschaffen haben, waren nämlich auch die lateinischen und die ihnen zugrundeliegenden griechischen Termini durchaus motiviert und damit "durchsichtig", sie sollten und konnten dem Lernenden zugleich ein stück Definition liefern, ihm mindestens das Behalten erleichtern.

Einige wenige Beispiele: Wenn man griechisch "rhema" und lateinisch "verbum" sagte, wollte man damit zugleich darstellen, daB die wörter dieses Typs, dieser Wortklasse eine besonders zentrale stellung einnehmen, daß sie gewissermaßen "Wörter als solche" sind. Wenn man griechisch "onoma" und lateinisch "nomen" sagte, wollte man damit die besondere Namen-Funktion der wörter dieser Wortklasse hervorheben. Wenn man lateinisch die beiden Arten der Nomen als "nomina substantiva" und "nomina adiectiva" unterschied (nämlich nach dem Kriterium, ob das betreffende Nomen ein bestimmtes grammatisches Geschlecht hatte oder je nach Bedarf 
alle drei grammatischen Geschlechter annehmen konnte), so wollte man mit dem Terminus "substantiva" diese wörter als "Namen von Wesen" kennzeichnen, mit dem Terminus "adiectiva" wollte man einen Hinweis auf die syntaktische Funktion dieser wörter geben, daß sie nämlich zu den substantiva hinzugefügt werden können und dabei je nach Bedarf verschiedene Formen (verschiedene Geschlechter, Singular oder Plural) annehmen.

17. Die Motiviertheit der lateinischen Termini für den heutigen Lernenden

Für den heutigen Lernenden ist die ursprüngliche Motiviertheit der lateinischen Termini nun keineswegs verbindlich. Die Termini "Verb" und "Adjektiv" wird er rein als Etiketten auffassen. Bei "Nomen" wird er evtl. aus der Nähe zu "Namen" eine gewisse Merkhilfe gewinnen. Bei "Pronomen" wird er aus dem Terminus die Merkhilfe gewinnen, daß diese wörter mit den Nomen zu tun haben, indem sie ein Nomen vertreten oder ein Nomen einleiten (z.B. oie, die Frau, diese Frau); man darf dann nur nicht die verengende Ubersetzung suggerieren "Fürwort", sondern muB den Namensbestandteil "Pro-", wenn man ihn schon erklären will, in seiner vollen, im Lateinischen vorhandenen Bedeutungsbreite vorstellen, nämlich als für wie als vor.

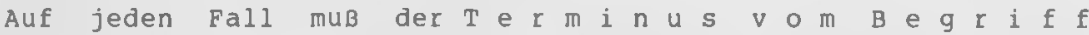
$h$ e $r \quad b$ e $l$ e $u$ c $h t$ e $t$ (und oft relativiert) werden, nicht etwa der Begriff vom Terminus her erschlossen. Ich betone daher hier ein weiteres Mal die Forderung (mit nochmaligem Hinweis auf den Beitrag von Elly Glinz, in welchem dazu Genaueres gesagt wird, speziell über die Lernprozesse bei kleinen Schülern): Wer sinnvoll über grammatische $T$ e $r m$ i $n$ reden will, muB zuyleich über die durch diese Termini zu bezeichnenden grammatischen $B$ e $g$ I $f$ f e reden, und er muB dabei immer die $A$ u $s$ w $i$ r k $n$ g e $n$ der Termini auf das $E$ r l e $r$ e $n$ de $r$ B e $g r$ i $f$ e - und zwar durch Lernende jedes Alters und jedes Begabungstyps - im Auge haben. Diese Forderung leitet hinüber zu einem kleinen geschichtlichen Rückblick. 
Die KMK-Liste von 1982 ist nicht der erste Versuch zu einer Vereinheitlichung der grammatischen Termini. Ihr sind schon 1961/65 die sogenannten "Hamburger Empfehlungen" vorausgegangen. An diesen Empfehlungen habe ich damals intensiv mitgearbeitet, und es ist vielleicht nicht abwegig - gerade im Blick auf die von Wimmer und andern aufgestellten Forderungen nach Transparenz bei derartigen Normierungsvorschlägen - wenn ich etwas über ihr zustandekommen berichte.

Wir haben damals nicht nur eine Terminologie-Liste gegeben, sondern sind ausgegangen von einem Uberblick über den sprachunterricht überhaupt und über die Rolle der Grammatik dabei. Es brauchte einiges, bis wir in diesen Grundfragen einen genügenden Konsens erreicht hatten; aber als wir einmal so weit waren, konnten wir auf dieser Grundlage in schlüssiger Weise die konkreten, nach schulstufen differenzierten terminologischen Vorschläge ausarbeiten, die dann (weil die meisten unserer Arbeitstagungen in Hamburg stattfanden) als "Hamburger Empfehlungen" bekannt wurden; der vollständige Text ist als Anhang $z u$ diesem Beitrag auf den S. 215-220 abgedruckt.

"Wir" - das war eine Runde von etwa 30 Frauen und Männern: Linguisten, Didaktiker der sprache und Literatur, Leute aus schulaufsicht und Lehrerfortbildung, auch eine Reihe von voll in der Schulpraxis stehenden Lehrern der verschiedenen schulstufen und Schulformen. Wir trafen uns erstmals, auf Einladung von Anne Banaschewski (Leiterin der Lehrerfortbildung Hamburg) und Werner Meyer (Lehrerfortbildung Hessen) zu einer dreitägigen Folge von Referaten und Diskussionen über Sprachunterricht, Gramatik und Terminologie im April 1961 in der Reinhardswaldschule bei Kassel. Die Referate wurden gehalten von Erika Essen vom studienseminar Marburg (bekannt durch ihre "Methodik des Deutschunterrichts" von 1956, seither viele Auflagen), von Hans Schorer von der PH Bonn (bekannt durch seine mit wiechmann zusammen entwickelten Sprachbücher "Lebendige sprache") und von mir (ich hatte damals den Lehrstuhl für deutsche sprache und ihre Didaktik an der PH Kettwig, die nachher nach Duisburg verlegt und dann in die Gesamthochschule Duishurg integriert wurde). 
Die Diskussionen waren sehr grundsätzlich und oft recht hart. Das begann schon mit der Frage, was unter "Sprachlehre" (welcher die ganze Tagung ja gelten solltel iberhaupt zu verstehen sei. Einige sträubten sich gegen meine nüchterne Feststellung, "Sprachlehre" sei einfach ein deutscher Terminus für "Grammatik", speziell für das an der Grammatik, was in den Schulen zu behandeln sei. Sie nahmen den Terminus (Erstbeleg 1619, bei Ratke) wörtlicher und verstanden darunter im Anschluß an Leo Weisgerber "alles, was dazu dient, den Sprachbesitz des Kindes zu bereichern, seiner Sprachfähigkeit ziel und Wege zur Betätigung zu weisen..." (nach Weisgerbers Artikel "Deutschsprachige Bildung" im Handbuch der Erziehungswissenschaft, Band IV/2, 1932,.64, 72 , $85 \mathrm{ffl}$.

Diese erste Sitzungsfolge fürte uns daher noch nicht zum ziel, sondern erst mitten in die Probleme hinein. Konkreteres erreichten wir an einer zweiten dreitägigen Sitzung im November 1962, in Hamburg. Wir fingen nicht mit den Gymnasien an, sondern mit der Basis des ganzen schulaufbaus, mit der Grundschule. Dabei fanden wir vor allem einen Konsens darüber, daß zuerst ein G e f $\ddot{i}$ h 1 für die grammatischen Phänomene (Sätze, Wörter, Wortarten usw.) zu entwickeln und zu festigen sei und im $A \mathrm{n} c \mathrm{~h} 1 \mathrm{u}$ B daran dann ein bewußtes Erkennen und systematisches Benennen, in einer der sprachlichen Entwicklung der schüler angepaßten, aber planvollen und auf wissenschaftliche Analyse gestüzten stufung.

An einer dritten Tagung, November 1963, wieder in Hamburg, wurden zur Klärung der konkreten Möglichkeiten und für direkteren Einblick in SchülerReaktionen drei Versuchsstunden gehalten, mit einem 6. Schuljahr, je etwa 60-90 Minuten, ohne strikte Begrenzung auf eine "Lektionslänge" von 45 Minuten. Ich machte den Anfang mit einer stunde iber den Satz als Einheit, den Aufbau von Sätzen aus Teilsätzen und die Verbindung von Grammatik und Lesen. Erika Essen behandelte das Thema "sätze beim kreativen schreiben" und zeigte dabei die Reaktion der schuler auf ihre "Satzfiguren". Wiechmann (als schulpraktischer Partner von Schorer) behandelte den Zusammenhang von Haupt- und Nebensatz, speziell Kausalsatz.

Es war ein nicht ganz geringes Risiko, so vor 30 Fachkollegen mit unbekannten Schilern zu arbeiten - vor allem fiir mich, da ich seit 1956 nicht mehr in der schule war, sondern nur gelegentlich Demonstrationsstunden durchfühte. Wir brauchten auch sehr viel zeit für die stunden und die anschließenden (manchmal bis ins Pedantische gehenden) Besprechungen. Ich dachte schon, wir hätten diese zeit besser für direkte Weiterarbeit an den schon vorhandenen Empfehlungs-Entwürfen verwendet, anstatt den Umweg uber die Demonstrationsstunden zu machen. Aber als wir dann endlich wieder zu diesen Empfehlungsentwürfen zurückkehrten, ging es auf einmal viel glatter als vorher, wir brachten Formulierungen und Forderungen durch, die vorher auf Widerstand gestoßen waren, und am Schluß dieser Tagung waren die Empfehlungen für alle neun schuljahre bereinigt in der Form, in der sie dann 1964 im "Wirkenden Wort" (14, S. 275-279) zu lesen waren. Der Weg uber die Schulstunden war kein Umweg, sondern der direkteste Weg zum Ziel gewesen.

Zwischenergebnisse waren ubrigens, vor allem von Werner Meyer, seit 1961 in pädagogischen Fachblättern mitgeteilt worden - wir arbeiteten grundsätzlich nicht hinter verschlossenen Türen. Auf einer abschließenden Tagung im Mai 1965 wurde das Ganze noch einmal überpruft und durch die zwei generellen Abschnitte "Grundsätzliches" und "Zum Problem der Benennungen" ergänzt (vollständige Publikation im "Wirkenden Wort", 1965, 15, 5. 405-410). 
Die Wirkung dieser Empfehlungen abzuschätzen kann hier nicht meine Aufgabe sein - schon weil sie in ihrem Kern auf Vorschläge von mir zurückgehen. 10

Die Aufnahme bei den Linguisten und Didaktikern wurde vielleicht negativ beeinflubt durch den Umbruch in den Auffassungen von Linguistik und sprachunterricht, den man grob mit der Jahreszahl 1968 verknüpfen kann. In der zweiten Hälfte der 60 er Jahre bildete sich für manche jüngere Linguisten und Didaktiker so etwas wie ein neues Lebensgefühl, und damit auch das Gefühl, jetzt beginne eine völlig neue zeit (gleichgültig ob im AnschluB an die generative Transformationsgrammatik von chomsky oder an die Sprachbarrierentheorie von Bernstein oder an die sprechakttheorie von Austin und Searle oder an die sprachauffassung des späten Wittgenstein oder an generelle politische Forderungen nach Emanzipation oder an noch andere strömungen - so verschieden und sich zum Teil gegenseitig ausschließend alle diese Ansätze oft auch sein mochten). Damit konnte auch das Gefühl verbunden sein, Leute wie Erika Essen, Schorer und Glinz, die mit Anne Banaschewski im Vorspann der "Empfehlungen" genannt waren (und hinter denen man, wenn auch großenteils zu Unrecht, immer auch Leo Weisgerber sah) gehörten nicht mehr der nun beginnenden neuen zeit an, sondern der zu Ende gehenden, überwundenen zeit; sie seien daher nicht mehr so ernst zu nehmen.

Immerhin kann gerade ein Vergleich mit der um 17 Jahre jüngeren KMK-Liste zeigen, wieviel von den damaligen vorschlägen (sowohl Begriffe wie Termini) sich doch ganz unbemerkt durchgesetzt hat. Im deutschsprachigen Teil der schweiz, wo das Jahr 1968 im Bereich von schule und wissenschaft viel weniger hohe wellen warf, sind manche Formulierungen aus den "Hamburger Empfehlungen" fast wörtlich in eine ganze Reihe von Lehrplänen aufgenommen worden (vor allem die stufung "Gefühl für ....- bewußt erkennen und benennen"). 
20. Ein stück selbsterfahrung für die Teilnehmer, anhand von "Satz und Proposition"

Die restliche für das Referat zur Verfügung stehende zeit benutzte ich für eine Art "Selbsterfahrungs-Versuch mit terminologischen Umstellungen" mit den Teilnehmern, indem ich zwei Begriffe vorfühte, die in dieser Fassung und mit dieser verwendung der Termini nicht allen Teilnehmern vertraut waren. Ich referiere daher jetzt nicht nur das, was ich an der Tagung vorlegte, sondern auch einiges aus der anschließenden Diskussion. zunächst die beiden Begriffe, für die ich die Termini "Satz" und "Proposition" verwende:

\section{S a $t z$}

Was im geschriebenen/gelesenen Text mit Grobbuchstaben anfängt und durch Punkt, Ausrufezeichen oder Fragezeichen mit nachfolgender Großschreibung abgeschlossen ist, grundsätzlich dem entsprechend, was im gesprochenen/gehörten Text unter e $i \mathrm{n}$ e m Melodiebogen steht und demgemäB, auch wenn es durch pausen unterteilt ist, als e i $n$ e Einheit für das zuhören hingestelt wird.

Dieser Begriff entspricht dem, was englisch "sentence", französisch "phrase", italienisch "periodo" (nicht mit der deutschen "Periode" zu verwechseln!) genannt wird.
P r o p o s i $t$ i o $n$

Was (gesprochen oder geschrieben, gehört oder gelesen) um ein einziges Verb oder Verbgefüge herum aufgebaut ist, d.h. was auf einer einzigen Verbalstruktur beruht o d e r

was als eigene Einheit ohne Verb dasteht und nicht einfach als satzglied in eine benachbarte Einheit mit Verb integrierbar ist.

Dieser Begriff entspricht im ersten Teil der Definition dem, was englisch als "clause", französisch als "proposition", italienisch als "proposizione" bezeichnet wird. Er ist aber insofern neu und weiter gefabt, als er auch die nichtverbalen selbständigen Gebilde umfabt (zweiter Teil der Definition).

Wenn man die beiden Begriffe in dieser Fassung verwendet, lassen sich über den Aufbau von Texten, gleichgültig welcher Art, die folgenden Aussagen machen:

a) $J$ e d e r Text besteht aus $p r \circ p \circ s i t i \circ n$, im Grenzfall aus einer einzigen Proposition (z.B. "In diesem Raum nicht rauchen"), und diese proposition kann im Grenzfall 
aus einem einzigen wort bestehen (z.B. "Nichtraucher", als Aufschrift). Diese generelle Aussage über Texte aller Art wird möglich, weil durch den zweiten Teil der Definition auch die genuin nichtverbalen Bestandteile von Texten erfast sind. Nach allen früheren satzdefinitionen müBte man sagen: Ein Text besteht aus Sätzen und/oder nicht satzartigen (nicht als Sätze zu anerkennenden) Gebilden.

b) In jedem $g$ e $s p r o c h$ e $n$ e $n$ Text teilt der sprecher durch seine stimmführung die propositionen $z$ u $g l$ e i $c h$ auch in $S$ ä $t z$ e und $T$ e $i s$ ä $t z e$ ein - wenn auch die Entscheidung, ob eine Proposition als ein satz oder als ein bloBer Teilsatz aufzufassen ist, beim Hörer nicht immer eindeutig getroffen werden kann (und auch nicht wichtig ist).

c) In $s$ c $h r i f t i d h$ e $n$ Texten stellt man in erster Linie die $p r o p \circ s i t$ o $n$ e $n$ dar, nämlich durch die wörter, die man schreibt. Meistens gibt man dazu, als Hilfe für die Leser, a u c h eine Einteilung in sätze/Teilsätze, durch die Verwendung der Satzzeichen (Punkt/Ausrufezeichen/ Fragezeichen mit nachfolgender Großschreibung als Signal für Satzende, dazu im Inneren der Sätze oft Komma, Doppelpunkt, Strichpunkt, Gedankenstrich, zwecks Binnengliederung, Einteilung in Teilsätze usw.). Es gibt aber auch gedruckte Texte, z.B. moderne Gedichte, ohne satzzeichen und ohne Grobschreibung des ersten Wortes eines Satzes. In solchen Texten ist also noch gar keine Einteilung in sätze gegeben, sie muB vom Leser geleistet werden, und sie kann von einem Leser zum andern verschieden ausfallen. Im Blick auf die Geschichte des Schriftwesens ist $z u$ sagen, daß bis zum Ende des Altertums fast alle (und noch bis zum Beginn des Buchdrucks sehr viele) Texte ohne Interpunktion geschrieben wurden, so daß es dem Leser überlassen war, wie er die Propositionen, die er aus den Wörtern entnahm, zu Sätzen zusammenfaßte. Das bedingte zugleich ein langsameres und intensiveres Lesen (und in der Regel ein lautes Lesen). Der zwang zu langsamerem Lesen und erhöhter Aufmerksamkeit ist wohl auch der Grund, warum gerade moderne Schriftsteller ihre Gedichte (und hie und da auch 
Prosastücke) manchmal ohne Satzzeichen und damit ohne festgelegte Einteilung in sätze drucken lassen.

Im Einzelnen kann man jeweils unterscheiden:

- Eine proposition kann z u g 1 e i c h ein satz sein, d.h. durch Punkt usw. abgeschlossen (oder umgekehrt gesehen, ein Satz kann aus einer einzigen proposition bestehen). Dann liegen e i $n$ f $c h$ e $s$ ä $t$ e vor:

Er weiß das. Und seine Kollegen wissen es auch. Das ist mir ganz klar.

- Eine proposition kann mit einer andern proposition oder mehreren andern $z u$ einem e $i n z$ i $g$ n satz zusammengenommen werden. Dann ist jede einzelne proposition ein T e i 1 $s$ a $t z$, und das Ganze ist ein Satz mit Teilsätzen:

Er weiß das, und seine Kollegen wissen es auch, das ist mir ganz klar.

Viele propositionen sind so gebaut, daß sie gleich gut als einfache Sätze oder als blobe Teilsätze gesetzt (gesprochen bzw. durch satzzeichen markiert) sein können. Andere sind so gebaut und aufeinander bezogen, nämlich als Hauptsätze und Nebensätze, daß man sie nur ausnahmsweise als einfache sätze hinstellt:

Das ist 80 , ich weiß es genau, weil er es mir selber gesagt hat.

Drei Propositionen, alle als Teilsätze gesetzt

Das ist so. Ich weiß es genau, weil er es mir selber gesagt hat.

Drei Propositionen, eine als einfacher Satz, zwei als Teilsätze gesetzt

Das ist so. Ich weiß es genau. Weil er es mir selber gesagt hat.

Nochmals die drei gleichen Propositionen, jetzt jede als einfacher satz

Die beiden Begriffe "Proposition" und "Satz" sind also immer eng aufeinander bezogen zu sehen - aber sie sind auch grundsätzlich zu unterscheiden. 
Der "Selbstversuch" für die Tagungsteilnehmer, als der diese Demonstration von "proposition und Satz" gedacht war, erfillte seinen zweck, die Schwierigkeiten beim Ubergang zu anderen begrifflichen und terminologischen Fassungen zu demonstrieren.

Einigen Teilnehmern stand deutlich, wie die Diskussion zeigte, beim Verständnis des hier vorgelegten Begriffs "proposition" der Begriff im Wege, der in der an logischen Formalismen ausgerichteten Linguistik mit dem Terminus "proposition" gemeint ist, nämlich der "abstrakte Satzinhalt", der als Verknüpfung von logischem subjekt und logischem prädikat bzw. als Kombination eines Arguments mit einer Funktion gesehen wird. Ein Beispiel wie Das war eine neue Erfahrung umfabt nämlich nach dieser logischen Definition zwei Propositionen (Das war eine Erfahrung die Erfahrung war neu), während es nach der von mir verwendeten Definition eine einzige proposition ist (nämlich auf der verbalen Wortkette "eine neue Erfahrung sein" beruht, mit dem subjekt das). Ein entsprechendes Textstück würde auch in der englischen Grammatik als eine einzige "clause" angesprochen, in der französischen Grammatik als eine einzige "proposition", in der italienischen Grammatik als eine einzige "proposizione".

Daß es anspruchsvoll sein kann, mit den beiden immer eng aufeinander zu beziehenden Begriffen "Satz" und "Proposition" stets klar und konsequent umzugehen, läßt sich auch an den Auszügen aus Quirk/Greenbaum (oben S. 30 und S. 32f.) beobachten, nämlich am Schwanken zwischen "sentence" und "clause", wo offenbar ein und dieselbe Konstruktion mit there is... gemeint ist.

21. Zum Abschluß nochmals: relative Unabhängigkeit des Begriffs von einem Terminus

Ein Vergleich der eben vorgeführten Unterscheidung "Satz und proposition" mit den diesbezüglichen Punkten aus den "Hamburger Empfehlungen" ( $\mathrm{s}$. Anhang I, S. 215-220) kann auch nochmals deutlich machen, $d a B$ eine begriffliche Unterscheidung vorhanden 
oder mindestens klar angebahnt sein kann, auch wenn kein Paar von einfachen Termini dafür eingeführt ist. Wenn in Punkt A6 die Rede ist vom "gliedmäßigen Aufbau des satzes mit einem verb als Kern und Rahmen und einer wechselnden Anzahl von Gliedern", ist damit klar das gemeint, was ich heute als "Proposition" bezeichne. Dasselbe gilt für den Punkt 35 und den Punkt C2 "Einsicht in den Bau und Ablauf des einfachen satzes, Betrachtung der verbalen Teile in ihrer stellung" usw. und "Einsicht in den Satzbau als Ausdruck einer geistigen ordnung, verbale Teile und Satzglieder" usw. Ebenso klar ist aus der gesamten Formulierung, daB in den Punkten Al und Bl nur das gemeint ist, was ich heute als "Satz" von der "Proposition" abhebe: "Gefühl für den Satz als inhaltliche Einheit (Satz = was in einem zug gesprochen wird..." usw.) und "Vertiefung des Gefühls für den Satz und seinen spannungsbogen als sinneinheit und Klanggestalt".

Es zeigt sich aber zugleich, welchen vorteil es bietet, wenn man nicht immer durch den umgebenden Text auf den jeweils gemeinten Aspekt eines mehrschichtigen Begriffs aufmerksam machen muB, sondern zwei eng miteinander zu verknüpfende Begriffe ansetzt und für jeden von ihnen einen besonderen Terminus einführt: Termini als $H i l$ f e $n$ für den Umgang mit den Begriffen, so wie generell in der sprache die $N$ a $m$ e $n$, die $w$ ö $t$ e $r$ als $\mathrm{H}$ i 1 f e $n$ dienen für den Umgang mit den Bedeutungen - mit hoher Schätzung dieser grundsätzlich unentbehrlichen Hilfen, aber ohne jede $U$ b e $r$ schätzung und mit wahrung des $P$ I $i$ or i$t$ ä $t$ s verhältnisses von der $B$ e $d$ e $u t u n g$ zum dafür vorhandenen oder neugebildeten $W \circ r$ und entsprechend vom $B$ $g$ I $f$ z zum dafür gewählten oder neugebildeten $T$ e $I m$ in $\mathrm{u} s$. 


\section{Anmerkungen}

1 So die lateinische Schulgrammatik in Deutschland im 18 . Jh. Nach: Jellinek, zweiter Halbband 1914, S. 466.

2 So Petrus Ramus, 1515-1572, in seiner grammatica, 1ib. III, cap. 2, zitiert bei Jellinek (1914) in der Anmerkung auf S. 466.

3 So Glinz (1952).

4 So Engel (1982) S. 178 und weiterhin.

5 Lateinische schulgrammatik in Deutschland im 18. Jh., Beleg bei Jellinek (1914) S. 466.

6 Nachdem ich vorher lange einen Terminus "Einreihgröße" überlegt hatte, um einen möglichst deutlichen Hinweis auf eine häufige Funktion dieses Satzglieds zu haben.

7 In der Duden-Grammatik (1959).

8 So Engel (1982) S. 185f, in der ersten Auflage S. 173f.. mit Kritik an der Namengebung von Glinz (1952) und Grebe in der Duden-Grammatik (1959).

9 In: Deutsche Grammatik II, S. 51-55.

10 Wer das nachprifen möchte, kann meinen Band: Sprachliche Bildung in der höheren Schule (1961, zweite Auflage unter dem Titel: Die Sprachen in der Schule, 1965) zur Hand nehmen und dort auf S. 5If. nachlesen, was (im Blick auf einen sachgerechten Übergang zum Lateinunterricht) für das Grammatikpensum der Grundschule vorgeschlagen wird.

\section{Literatur}

Drach, E. (1937): Grundgedanken der deutschen Satzlehre, Frankfurt/Main. Duden (1959): Grammatik der deutschen Gegenwartssprache, Mannheim.

Enge1, U. (1982): Syntax der deutschen Gegenwartssprache, 2. Uiberarbeitete Auflage. Berlin (1. Auflage 1977).

Erdmann, P. (1983): Zur 'Existenzialität' von there-Sätzen im Englischen. In Zeitschrift für Sprachwissenschaft 2,2. S. 220-232.

Glinz, H. (1952): Innere Form des Deutschen, Bern.

Glinz, H. (1961): Sprachliche Bildung in der Schule. Duisseldorf.

Glinz, H. (1971): Deutsche Grammatik II. Kasussyntax-NominalstrukturenWortarten-Kasusfremdes. Wiesbaden.

Jellinek, M.H. (1914): Geschichte der neuhochdeutschen Grammatik von den Anfängen bis auf Adelung. Zwei Halbbände. Heidelberg.

Jespersen, Otto (1924): The Philosophy of Grammar. London.

KMK-Liste (1982): Sekretariat der Ständigen Konferenz der Kultusminister der Länder in der Bundesrepublik Deutschland. Verzeichnis grundlegender grammatischer Fachausdricke.

Polenz, P. von (1969): Der Pertinenzdativ und seine Satzbaupläne. In: Engel, U., Grebe, P., Rupp, H. (Hrsg.), Festschrift für Hugo Moser zum 60 . Geburtstag. Disseldorf, S. 146-171.

Quirk, R./Greenbaum, S. (1973) : A University Grammar of English. London.

Scott, F.S. et al. (1968): English Grammar. A Linguistic study of ist Classes and Structures. London, Auckland.

Thomson,/Martinet, A. (1979): A Practical English Grammar. 2 nd ed.London. Wimmer, R. (1983) : IdS-Stellungnahme zu dem "Verzeichnis grundlegender grammatischer Fachausdricke (in der Kultusministerkonferenz zustimmend zur Kenntnis genommen am 26.2.1982). In: Mitteilungen des Instituts für deutsche sprache 9, s. 5-30. 